\title{
The Association between Adiposity and the Risk of Glaucoma: A Meta-Analysis
}

\author{
Weiming Liu, ${ }^{1}$ Jiawen Ling, ${ }^{2}$ Yiyi Chen, ${ }^{1}$ Yan $W u,{ }^{1}$ and Peirong $\mathrm{Lu}^{1}$ \\ ${ }^{1}$ Department of Ophthalmology, The First Affiliated Hospital of Soochow University, 188 Shizi Street, Suzhou 215006, China \\ ${ }^{2}$ Department of Ophthalmology, The Third People's Hospital of Zhangjiagang, Zhangjiagang, China
}

Correspondence should be addressed to Peirong Lu; lupeirong@suda.edu.cn

Received 10 February 2017; Revised 6 May 2017; Accepted 18 May 2017; Published 12 June 2017

Academic Editor: Jesús Pintor

Copyright (C) 2017 Weiming Liu et al. This is an open access article distributed under the Creative Commons Attribution License, which permits unrestricted use, distribution, and reproduction in any medium, provided the original work is properly cited.

\begin{abstract}
Purpose. This meta-analysis was conducted to determine the potential association between adiposity and glaucoma incidence. Materials and Methods. A comprehensive literature search was performed in PubMed and ISI Web of Science. A meta-analysis was conducted using STATA software. Results. Fifteen eligible studies involving 2,445,980 individuals were included to investigate the association between adiposity and glaucoma incidence. The relative risks (RRs) were pooled with $95 \%$ confidence intervals (CI) by using a random-effects model. The pooled RR between adiposity and elevated intraocular pressure (IOP) was 1.73 (95\% CI, 1.18-2.54), whereas that between adiposity and open-angle glaucoma (OAG) was 0.97 (95\% CI, 0.83-1.13). The pooled RR between abdominal adiposity and glaucoma was 1.28 (95\% CI, 1.15-1.41), whereas that between general adiposity and glaucoma was 1.09 (95\% CI, 0.87-1.37). Results of subgroup analysis by sex indicated the association between adiposity and glaucoma in the female group (RR, 1.31; 95\% CI, 1.05-1.64), but not in the male group (RR, 1.11; 95\% CI, 0.77-1.60). The pooled RR of cohort studies and cross-sectional studies were 1.00 (95\% CI, 0.84-1.20) and 1.22 (95\% CI, 0.89-1.66), respectively. Conclusions. Adiposity has a higher risk of elevated IOP, and abdominal adiposity has a positive association with glaucoma, especially in female patients.
\end{abstract}

\section{Introduction}

Glaucoma is the first leading cause of irreversible blindness [1]. OAG is the most common type of glaucoma [1]. The main risk factor for OAG is considered to be elevated IOP [2] and other systematic risk factors, including older age, family history of OAG [2], diabetes mellitus [3], alcohol consumption [4], hypertension, and cigarette smoking [5].

Globally, being overweight and adiposity pose a threat to children and adolescents both in developed and developing countries. However, whether anthropometric factors, such as general adiposity or abdominal adiposity, are determinants of OAG or elevated IOP risk is still unclear.

To assess body-weight status, body mass index (BMI) is usually used as an indicator of general adiposity, and waist circumference (WC) or waist-to-hip ratio (WHR) is used for abdominal adiposity [6]. Previous studies [7, 8] have found that a higher BMI is related to glaucoma incidence, while some studies [2,9] did not report a positive association between BMI and elevated IOP or OAG. Other studies have shown positive trends and significant correlations between higher WC or WHR and elevated IOP $[10,11]$. These inconsistent results prompted this meta-analysis to provide a more accurate estimate of the association between adiposity and glaucoma incidence.

\section{Materials and Methods}

2.1. Search Strategy. This meta-analysis was conducted under the guidance of PRISMA [12]. A systematic search was carried out in PubMed and ISI Web of Science before December 2016, using the following terms: ((metabolic syndrome) OR (overweight) OR (obesity) OR (adiposity) OR (body mass index) OR (BMI) OR (intra-abdominal fat) OR (waist hip ratio) OR (waist circumference) OR (Anthropometric)) AND ((glaucoma) OR (intraocular pressure) OR (ocular 
hypertension) OR (open-angle glaucoma) OR (normal tension glaucoma) OR (high tension glaucoma)). The search was run according to Medical Subject Headings (MeSH), without restriction to regions, or publication types. The language was restricted to English. Citations for related articles were detected for additional publications. Where several reports related to the same study, only the most recent report was used.

2.2. Inclusion and Exclusion Criteria. The published study was included in this meta-analysis if it met all the following criteria: (1) reports the association of adiposity, BMI, WC, or WHR with glaucoma, or elevated IOP; (2) adopts a cohort, case-control or cross-sectional design; (3) stratifies BMI, WC, or WHR into more than two stratifications; and (4) presents the $\mathrm{RR}$, odds ratio (OR), or original data that could calculate $\mathrm{RR}$ values.

Studies were excluded if any of the following criteria were identified: (1) studies were case reports or case series; (2) studies were not conducted in human adults; (3) studies were conducted in population samples comprising only patients with obesity, metabolic syndrome, glaucoma, or OHT at baseline; (4) studies selected close-angle glaucoma as an outcome; and (5) studies concerned drug effects or specific conditions (e.g., eye surgery).

2.3. Data Extraction and Assessment of Study Quality. Data were extracted and summarized from all eligible studies by two independent reviewers (Weiming Liu and Yiyi Chen). Any disagreements were discussed by the two reviewers or resolved by adjudicating senior authors (Peirong Lu). The data included the following: name of the first author, publication year, study design, study follow-up period, country, database of the data collection from, number of participants (case/control), age, outcome definition, exposure definition, adjustment factors, and OR/RR value with a 95\% CI.

Because there is no suitable standardized assessment method to assess the quality of observation studies, including cohorts, case-control, and cross-sectional design, for this meta-analysis, a quality assessment tool was designed according to MOOSE, STROBE, and references [13-16]. Two independent reviewers who were blind to each other (Weiming Liu and Yan $\mathrm{Wu}$ ) assessed the quality scales and resolved any disagreements through discussion with senior authors (Jiawen Ling and Peirong Lu). The studies which scored eight or greater on quality scales were considered to be of a relatively high methodological quality. The detail of the items and the points of each study get are shown in Table 1.

2.4. Statistical Methods for the Meta-Analysis. For metaanalysis, RR with 95\% CI was assessed to determine the relationship between adiposity and glaucoma incidence. Adjusted data were used to assess the relationship between adiposity and the risk of glaucoma if the adjusted and unadjusted data were reported in the articles. When the results were provided by gender, the results were summarized into a single RR with a 95\% CI, using the fixed-effects method and under the assumption that OR were accurate approximations of RR [17].
Subgroup analyses were carried out according to adiposity measurement (BMI group or abdominal adiposity, including WC or WHR) and outcome definition (IOP group, or glaucoma group). In the exposure-definition subgroups, articles were divided into general adiposity (BMI) group or abdominal adiposity group (WC or WHR); the association between general or abdominal adiposity with glaucoma (including OAG or elevated IOP as outcome) was then assessed. In the outcome-definition subgroup, articles were divided into the IOP group or open-angle glaucoma group; the relationship between adiposity anthropometric stratification and IOP change or prevalence of OAG were then assessed. With the exception of the exposure-group analysis, it was preferable to use RR values on WC or WHR rather than on BMI.

Statistical heterogeneity was evaluated across studies using the $Q$ test and $I^{2}$ tests, where $P_{Q}<0.1$ or $I^{2}>50 \%$ represented significant heterogeneity across studies. Accordingly, the random-effects method was used to evaluate the potential relationship between adiposity and glaucoma for all analyses [18].

Moreover, sensitivity analyses were examined by deleting each study individually to evaluate the quality and consistency of the results. A series subgroup analysis was also conducted. Begg's test and Egger's test were used to evaluate the potential publication bias, and funnel plots were presented visually $[19,20]$. All statistical analyses were carried out using the STATA software package (version 12.0; STATA Corp., College Station, TX).

\section{Results}

3.1. Identification and Selection of Studies. Initially, 1161 articles were identified, comprising 644 from PubMed and 517 from ISI Web of Science. Among these articles, there were 264 duplicates and 852 unrelated articles, which were excluded. After reading the 45 full-text articles, 30 articles were excluded because they did not provide available data on BMI, WC, or WHR stratification. Ultimately, to conduct this meta-analysis, 15 studies were identified, which had been published from 1995 to 2016. A flowchart for the literature search work and results is shown in Figure 1.

3.2. Study Characteristics and Quality Assessment. A total of 2,445,980 individuals from all included studies were included. Table 2 showed the characteristics of 15 studies. In all, it was possible to identify nine cross-sectional studies $[2,8,10,11,21-25]$, one case-control study [26], and five cohort studies [7, 27-30]. The geographic distribution of these studies was six in the America [2, 7, 22, 28-30], one in Europe [27], seven in Asia [8, 10, 11, 23-26], and one in Africa [21]. The longest study period was more than 24 years [29], and study periods were different between the included studies.

Adjusted factors differed between the included studies, such as age, sex, alcohol consumption, smoking status, physical activity, hypertension, and diabetes. The quality scale for 11 of the studies was 8 or greater, which is considered to indicate a relatively high methodological quality, 


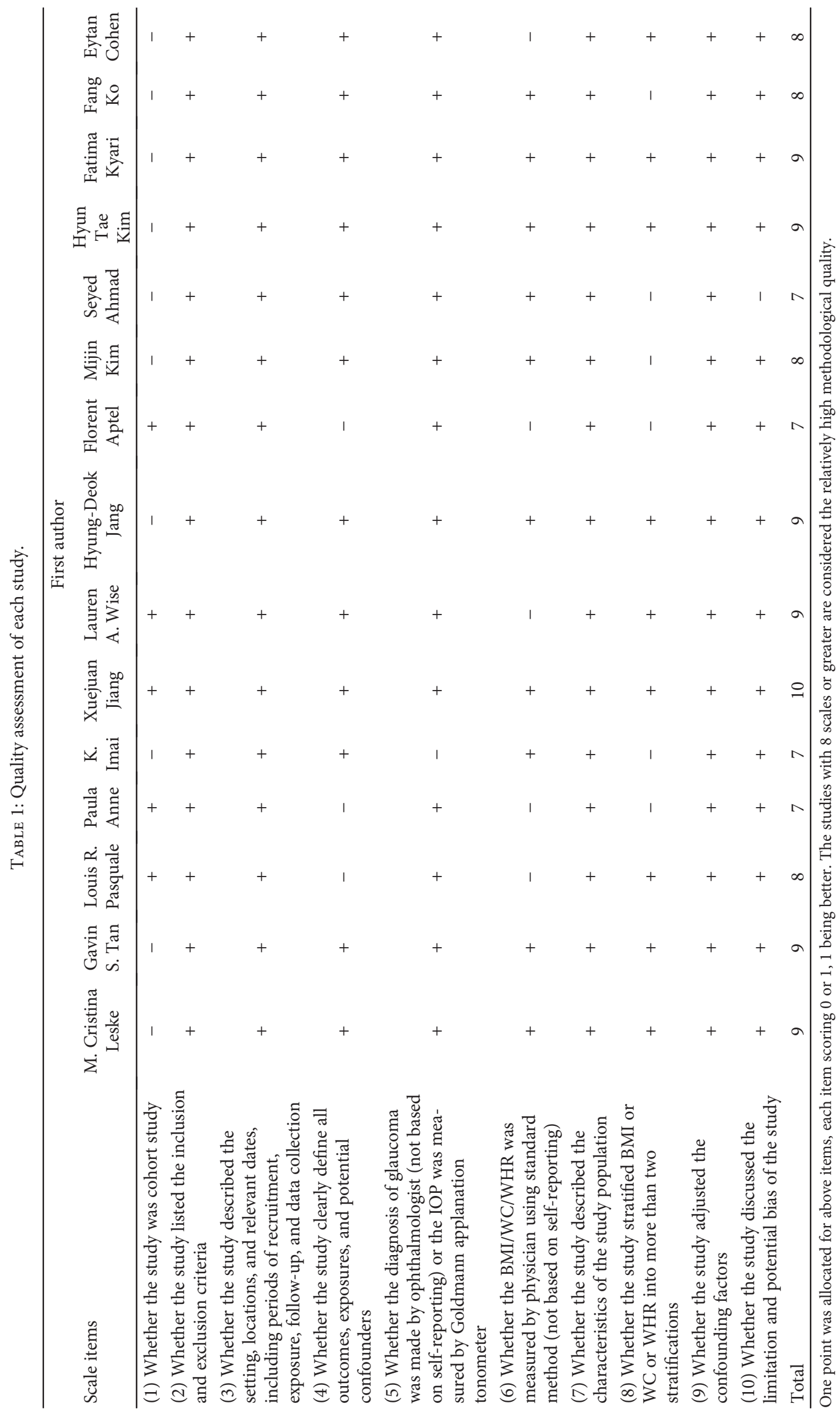




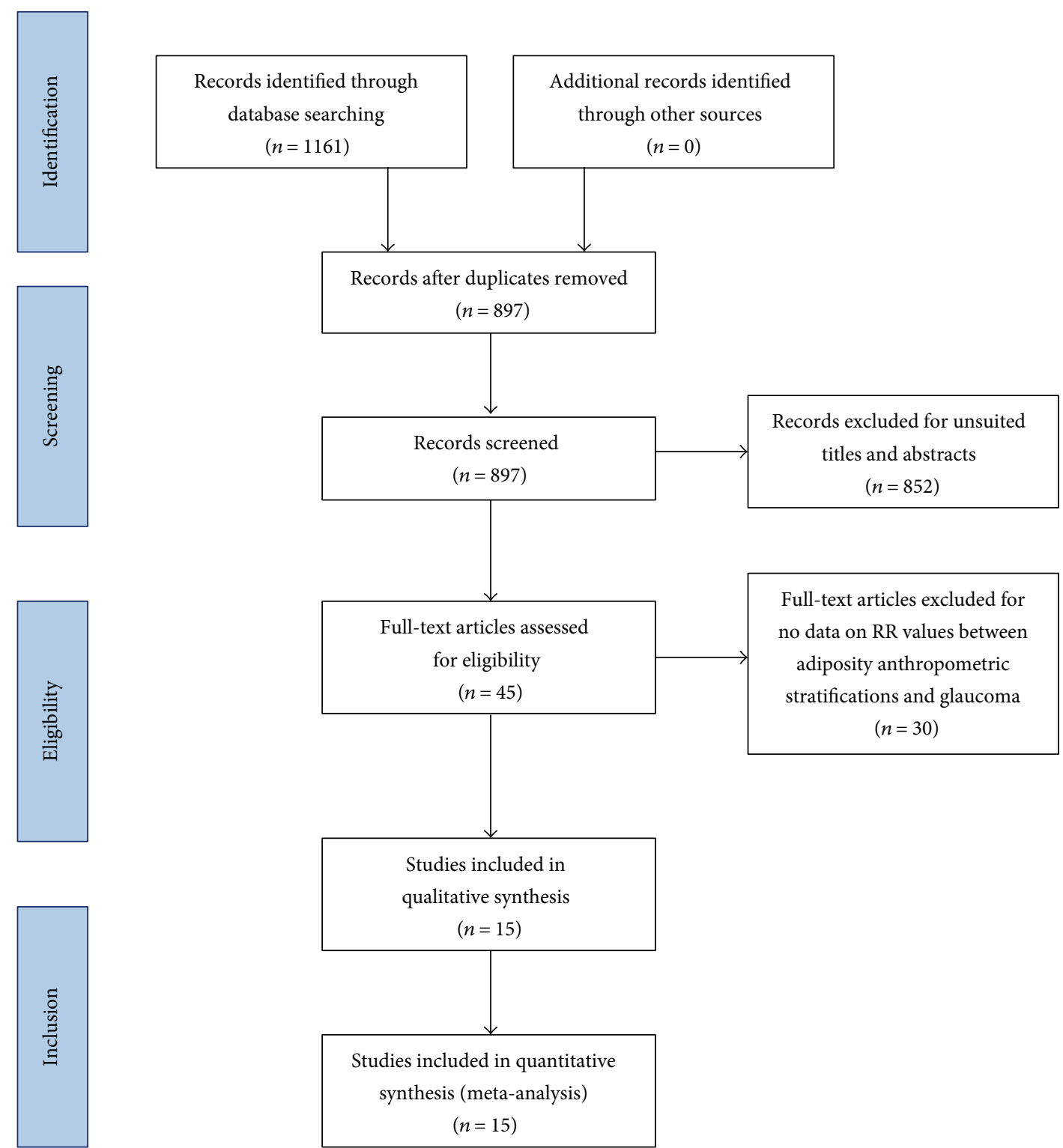

Figure 1: Flow diagram showing the selection process for inclusion of studies.

and the remaining 4 studies scored less than 8 (the average scale of 15 studies was 8.3 ).

\subsection{Pooled-Analysis Results}

3.3.1. Elevated IOP or OAG Group Analysis. The pooled RR for four studies using exclusively elevated IOP $[8,10,11$, 25 ] as an outcome was 1.73 (95\% CI, 1.18-2.54, $P=0.005$; $I^{2}=89.1 \%, P_{\text {heterogeneity }}<0.001$; Figure 2$)$, whereas the RR for 11 studies using OAG $[2,7,17-20,22-26]$ as an outcome was 0.97 (95\% CI, $0.83-1.13, P=0.709 ; I^{2}=78.9 \%, P_{\text {heterogeneity }}$ $<0.001$; Figure 2).

3.3.2. General or Abdominal Adiposity Group Analysis. The pooled RR for studies using exclusively abdominal adiposity measured by WC or WHR [7, 10, 11, 22, 24-26, 28] for exposure was 1.28 (95\% CI, $1.15-1.41, P<0.001 ; I^{2}=20.5 \%$, $P_{\text {heterogeneity }}=0.267$; Figure 3$)$; the heterogeneity was statistically insignificant, whereas the RR for studies using general adiposity measured by BMI $[2,7,8,10,11,21-23$, 27-30] for exposure was 1.09 (95\% CI, 0.87-1.37, $P=0.433$; $I^{2}=91.2 \%, P_{\text {heterogeneity }}<0.001$; Figure 3$)$.

3.3.3. Subgroup Analysis. The series subgroup (Table 3) that was conducted included study design, gender, smoking, alcohol intake, physical activity, hypertension, diabetes mellitus, other metabolic syndrome components, and central corneal thickness (CCT). Results of subgroup analysis by the study design did not indicate the significant association in the cohort study group (RR, 1.00; 95\% CI, 0.84-1.20; $\left.I^{2}=84.1 \% ; P<0.001\right)$ or in the cross-sectional study group (RR, 1.22; 95\% CI, 0.89-1.66; $I^{2}=88.6 \% ; P<0.001$ ). Because there was only one case-control study, it was not included in this subgroup analysis. In the gender subgroup analysis, the pooled RR for men $[2,8,10,25,29,30]$ was 1.11 (95\% CI, $\left.0.77-1.60 ; I^{2}=91.8 \% ; P<0.001\right)$, while the pooled RR for 


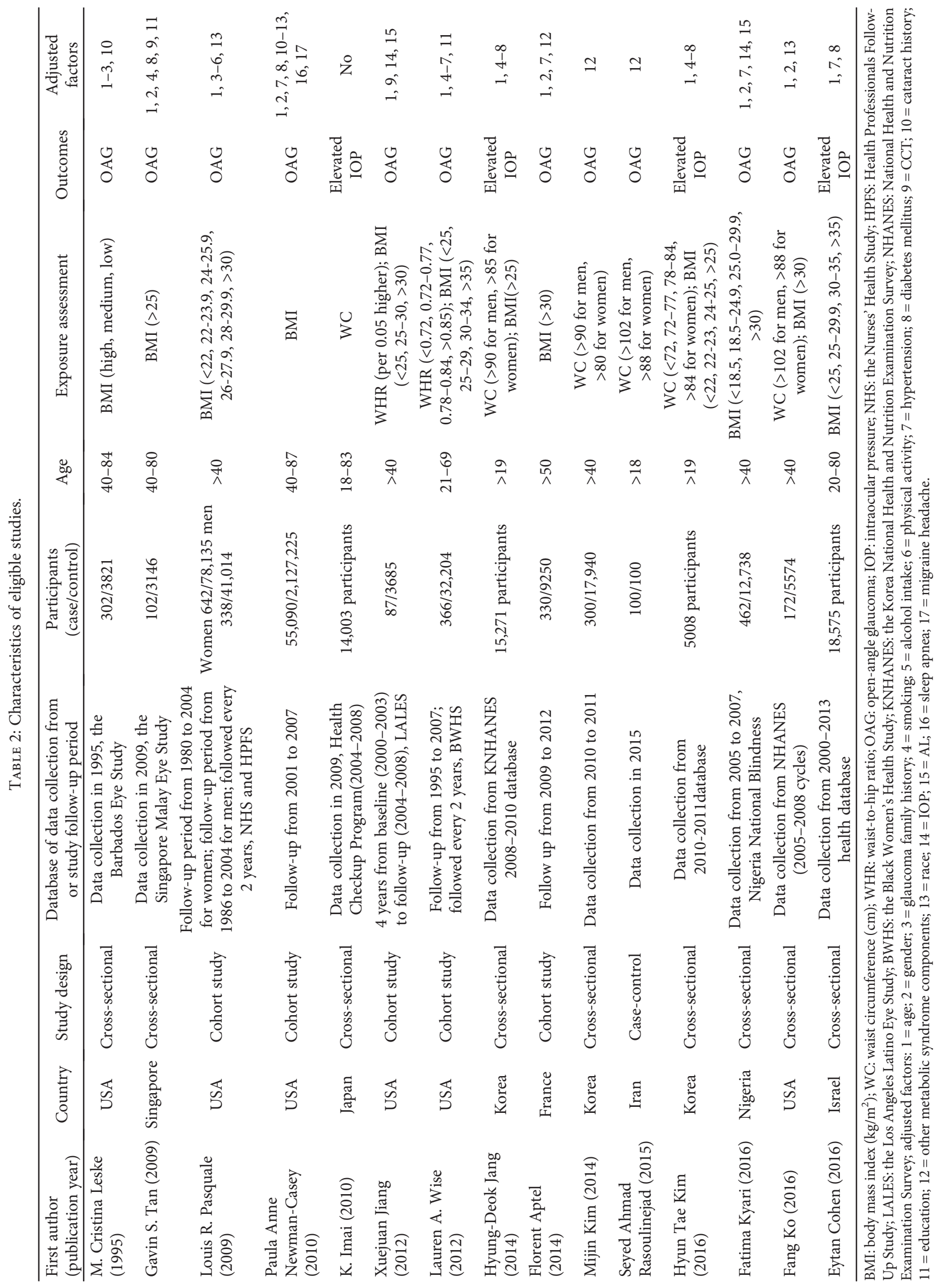




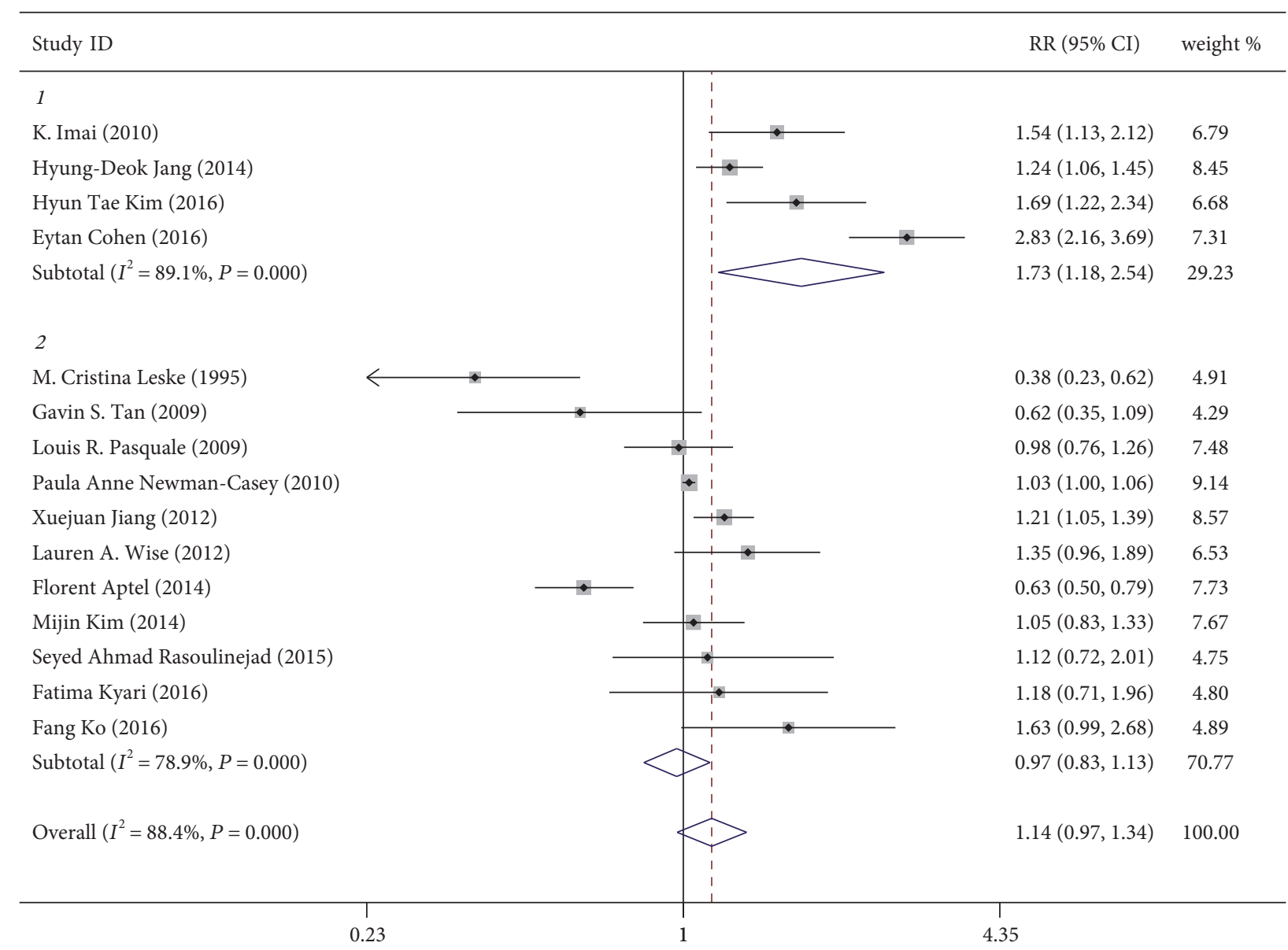

FIGURE 2: Forest plot for the association between adiposity and elevated IOP or OAG incidence. $1=$ elevated IOP group; $2=$ OAG group. Note: weights are from random-effects analysis.

women $[2,7,8,10,11,25,29,30]$ was $1.31(95 \% \mathrm{CI}, 1.05-$ $\left.1.64 ; I^{2}=80.3 \% ; P<0.001\right)$.

3.4. Sensitivity Analysis and Publication Bias. In the analysis, a sensitivity analysis was carried out to evaluate the stability of the results by deleting one study at a time and calculating the pooled OR for the remaining studies. Apart from Leske et al. [2] (pooled RR, 1.21; 95\% CI, 1.03-1.41) and Aptel et al. [27] (pooled RR, 1.20; 95\% CI, 1.02-1.41), when any other study was excluded, the estimated pooled RR was similar to previously (Figure 4). Begg's funnel plot and Egger's test revealed the absence of publication bias (Figure 5). The $P$ value for Begg's test was 0.843; for Egger's test, it was 0.383 .

\section{Discussion}

First, a meta-analysis was conducted to summarize the evidence from all available retrospective and prospective studies in order to evaluate the association between adiposity and the risk of elevated IOP or OAG incidence. Importantly, pooled data were provided for a substantial number of cases and controls to enable better understanding of this relationship. In this study, the pooled RR value suggested that adiposity had a positive association with the risk of elevated IOP, while there is no significant association between adiposity and OAG.
Second, a series of subgroup and sensitivity analyses were conducted, according to the anthropometric-parameter measurements of adiposity, as well as exposure stratification. A slight positive association between abdominal adiposity measured by WC or WHR and risk of glaucoma was found in this analysis; however, the relationship was insignificant between general adiposity measured by BMI with glaucoma incidence. Finally, it was possible to identify a relationship in the gender subgroup showing that adipose women had a higher risk of glaucoma than adipose men.

In previous studies, several theories explain the relationship between adiposity and glaucoma. One theory suggests that cerebrospinal fluid pressure (CSFP) and glaucomatous optic neuropathy may be due to either an elevated IOP, an abnormally low orbital CSFP, or higher translamina cribrosa pressure difference (TLCPD) [31, 32]. Obese patients have higher cerebrospinal fluid pressure, which may be related to a larger neuroretinal rim area equivalent to the optic nerve fibers $[33,34]$. Some studies suggested that taller body height with higher CSFP and lower TLCPD resulted in a lower prevalence of OAG [32]. However, other studies suggested that a taller person with a lower BMI has a higher risk of a smaller neuroretinal rim area and a larger optic cup-to-disc area ratio. Similarly, a taller person with a lower BMI may have a higher risk for developing OAG, while a higher BMI may 


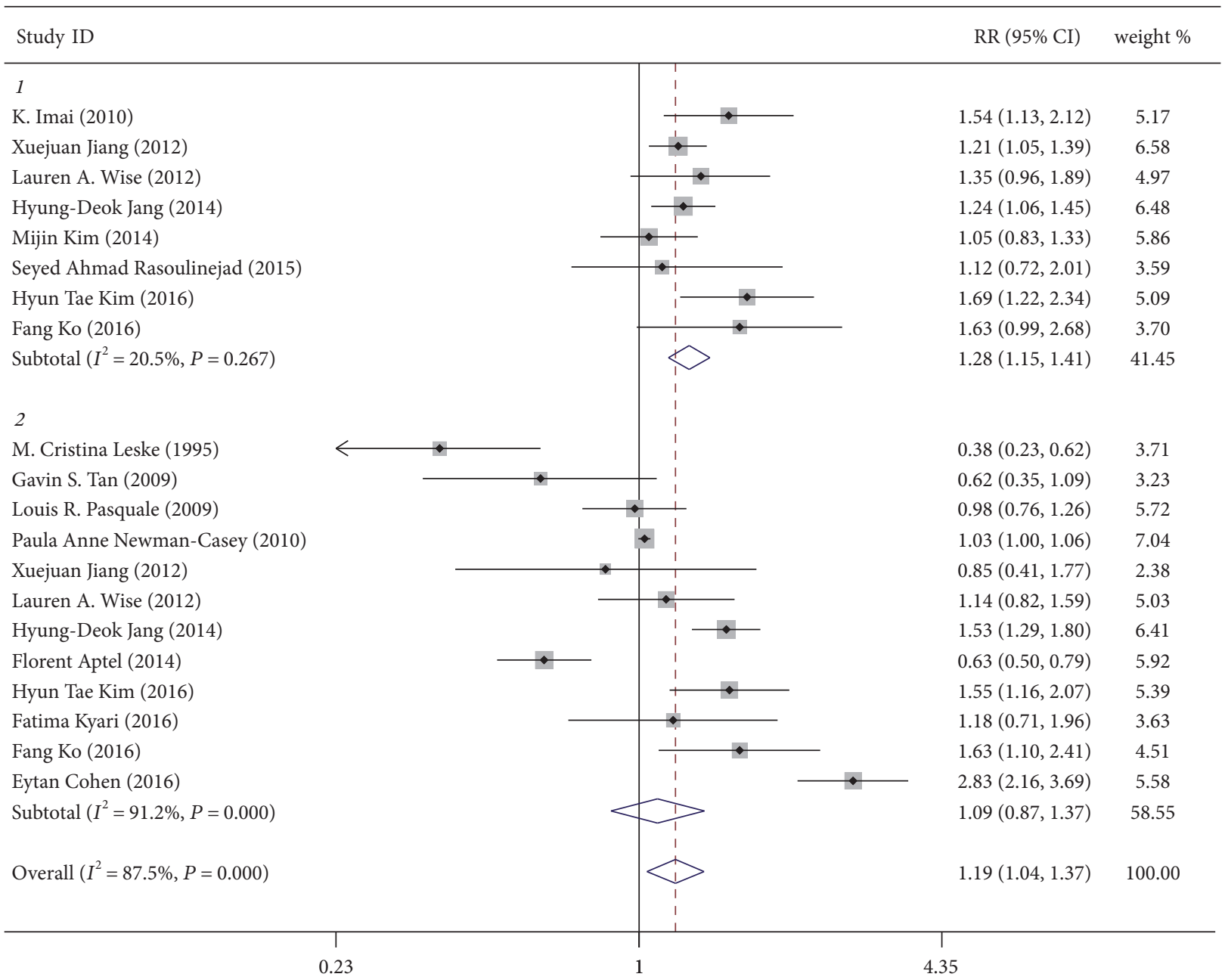

FIGURE 3: Forest plot for the association between general or abdominal adiposity and glaucoma. $1=$ abdominal group (measured by waist circumference or waist-to-hip ratio); 2 = general group (measured by body mass index). Note: weights are from random-effects analysis.

be a protective risk $[35,36]$. These findings suggest possible biological mechanisms for the pathogenesis of OAG, but more studies are needed to research the association between adiposity and CSFP and TLCPD, which are related to the risk of glaucoma.

The second hypothesis proposes that excess orbital fat tissue may increase episcleral venous pressure and blood viscosity, with increased outflow resistance in the episcleral veins, which could cause a decreased outflow facility and an increased IOP [37]. Moreover, obese patients may have a narrower orbital optic nerve subarachnoid space, which suggests a lower orbital CSFP involved in the pathogenesis of glaucoma [38]. In addition, there is a theory that an accumulation of lipid depositions may reduce the facility of aqueous outflow and secondarily elevate IOP [39]. Higher IOP and lower anterior chamber depth (ACD) would be significantly related to obesity [40].

Another possible mechanism supporting the association between adiposity and IOP is that hyperleptinemia, which accompanies obesity, may result in increased oxidative stress [41]. Compared to healthy subjects, the trabecular meshwork of obese patients with OAG has higher oxidative damage
[42]. Physical fitness has been shown to decrease IOP temporarily. Exercise has an effect on decreasing IOP because of lower norepinephrine concentrations, increased colloid osmotic pressure, coaction of nitric oxide, endothelin, and a $\beta 2$-adrenergic receptor gene polymorphism [43, 44]. Almost all obese patients with glaucoma and elevated IOP possibly are related to this mechanism because most obese patients undertake little exercise.

Furthermore, another study has suggested that the Valsalva maneuver, or breath-holding and thorax compression, may cause transitory elevation in IOP when obese patients were measured using the Goldmann tonometry $[45,46]$. Although the aforementioned mechanisms may be the cause of increased IOP elevation in obese patients, further studies are still required to better understand the mechanisms.

It was also found that abdominal adiposity measured by WC or WHR had a slight positive association with glaucoma and elevated IOP. However, these results have not been found in general adiposity measured by the BMI subgroup. This finding perhaps resulted from the use of different anthropometric methods. BMI was calculated as body 
TABLE 3: Results of subgroup analysis between adiposity and glaucoma with pooled RR.

\begin{tabular}{|c|c|c|c|c|c|}
\hline & Subgroups & Number of studies & RR (95\% CI) & $I^{2}, \%$ & Heterogeneity $P$ value \\
\hline \multirow{2}{*}{ Study design } & Cohort & 5 & $1.00(0.84-1.20)$ & $84.10 \%$ & $<0.001$ \\
\hline & Cross-sectional & 9 & $1.22(0.89-1.66)$ & $88.60 \%$ & $<0.001$ \\
\hline \multirow{2}{*}{ Gender } & Male & 6 & $1.11(0.77-1.60)$ & $91.80 \%$ & $<0.001$ \\
\hline & Female & 8 & $1.31(1.05-1.64)$ & $80.30 \%$ & $<0.001$ \\
\hline \multirow{2}{*}{ Smoking } & Yes & 5 & $1.18(0.93-1.48)$ & $68.20 \%$ & 0.014 \\
\hline & No & 10 & $1.13(0.90-1.41)$ & $91.20 \%$ & $<0.001$ \\
\hline \multirow{2}{*}{ Alcohol intake } & Yes & 4 & $1.26(1.04-1.54)$ & $57.60 \%$ & 0.069 \\
\hline & No & 11 & $1.09(0.87-1.35)$ & $90.50 \%$ & $<0.001$ \\
\hline \multirow{2}{*}{ Physical activity } & Yes & 4 & $1.26(1.04-1.54)$ & $57.60 \%$ & 0.069 \\
\hline & No & 11 & $1.09(0.87-1.35)$ & $90.50 \%$ & $<0.001$ \\
\hline \multirow{2}{*}{ Hypertension } & Yes & 7 & $1.28(0.98-1.69)$ & $93.20 \%$ & $<0.001$ \\
\hline & No & 8 & $1.02(0.80-1.29)$ & $77.50 \%$ & $<0.001$ \\
\hline \multirow{2}{*}{ Diabetes mellitus } & Yes & 5 & $1.35(0.96-1.91)$ & $94.30 \%$ & $<0.001$ \\
\hline & No & 10 & $1.03(0.83-1.29)$ & $82.20 \%$ & $<0.001$ \\
\hline \multirow{2}{*}{ Other metabolic syndrome components } & Yes & 4 & $0.92(0.72-1.18)$ & $82.60 \%$ & 0.001 \\
\hline & No & 11 & $1.23(0.98-1.56)$ & $86.30 \%$ & $<0.001$ \\
\hline \multirow{2}{*}{ CCT } & Yes & 2 & $0.92(0.48-1.75)$ & $80.10 \%$ & 0.025 \\
\hline & No & 13 & $1.17(0.97-1.41)$ & $89.40 \%$ & $<0.001$ \\
\hline
\end{tabular}

RR: relative risk; CI: confidence interval; CCT: central corneal thickness.

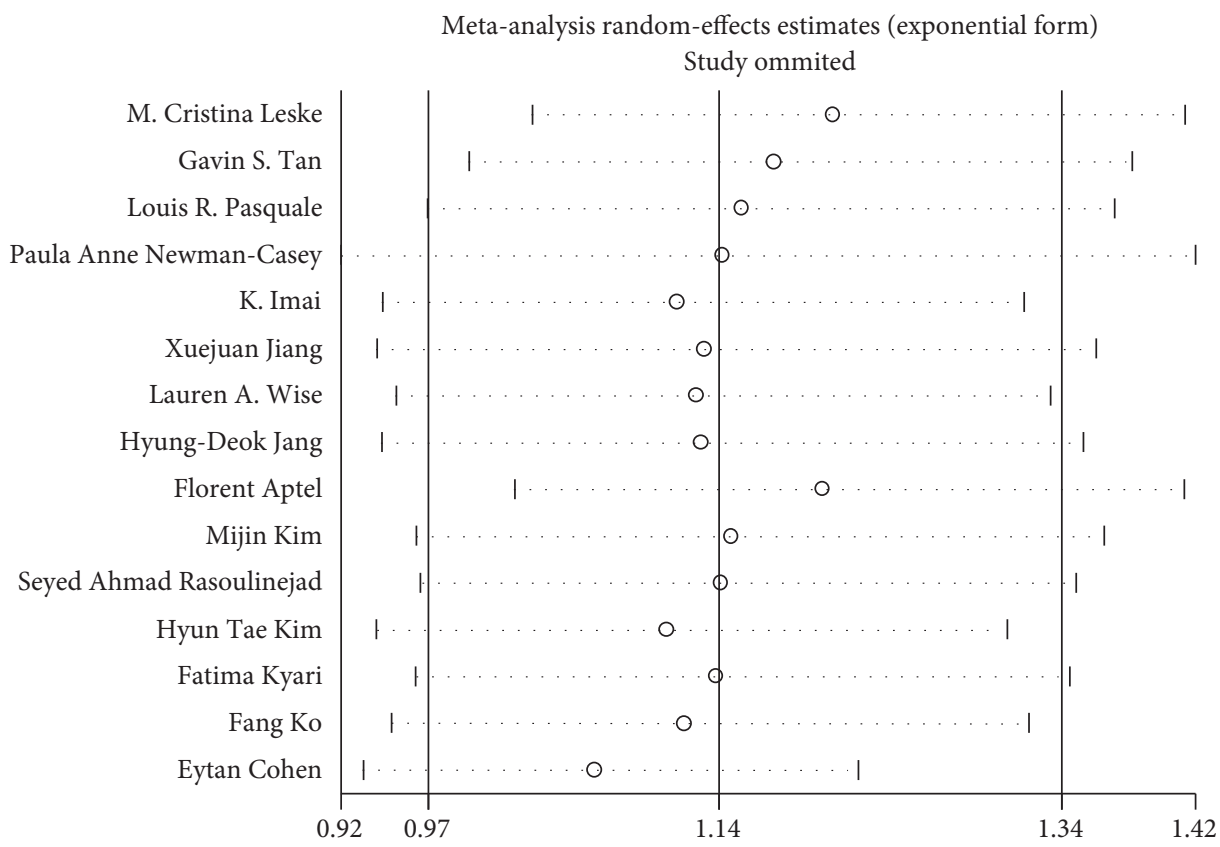

FIgURE 4: Sensitivity analysis of the association between adiposity and glaucoma.

weight $/$ height ${ }^{2}\left(\mathrm{~kg} / \mathrm{m}^{2}\right)$ and categorized as follows: normal weight $(\mathrm{BMI}=18.5-24.9)$, overweight $(\mathrm{BMI}=25.0-29.9)$, and obese $(\mathrm{BMI} \geq 30)$ [47]. Ethnic-specific waist circumference cut-offs have been incorporated into the definition. In Asian people, the relevant WC is above $90 \mathrm{~cm}$ in men and $80 \mathrm{~cm}$ in women, and in European people, it rises to above $94 \mathrm{~cm}$ in men and $80 \mathrm{~cm}$ in women [48]. Although BMI is used widely, it cannot measure adiposity parameters. Abdominal obesity plays an important role in the metabolic syndrome, and WC rather than BMI is recommended as the measurement [49].

WHR is a relatively new anthropometric index that is normalized by body size, which was proposed in the 1990s [50]. WHR is demonstrated as a better predictor of disease 


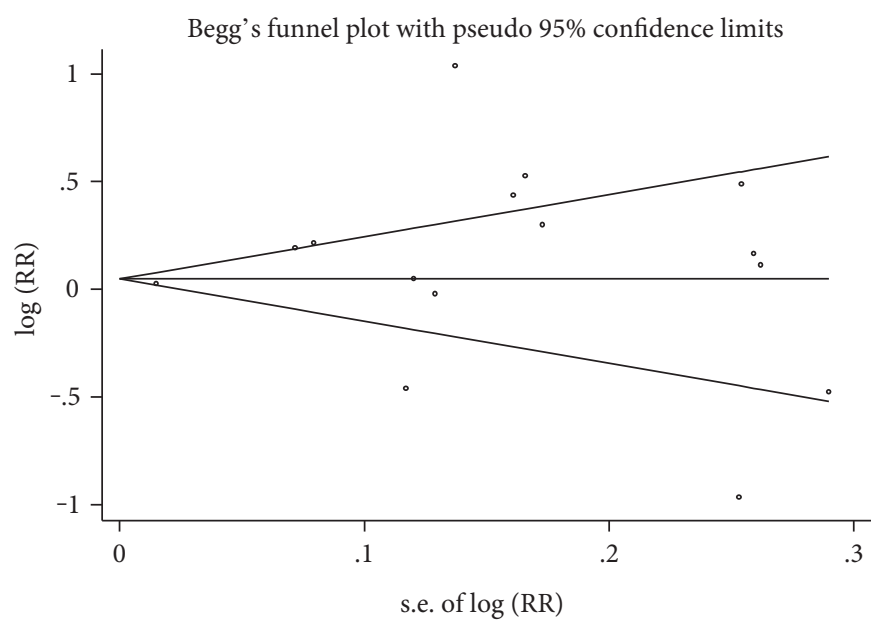

FIgURE 5: Funnel plot for studies of the association between adiposity and glaucoma.

or mortality risk than BMI [51], and it may be more related to age-related diseases, since muscle loss and changes in regional adipose tissue distribution are common with aging [52]. Among several anthropometric parameters, greater WC or WHR was correlated with higher IOP, but this association was of little clinical significance. WC or WHR is recognized as a good predictor of abdominal adiposity, while BMI does not reflect actual body condition. Moreover, Kim et al. also found that other adiposity parameters were related to elevated IOP, such as a higher fat ratio and lower lean body mass (muscle mass) and bone-mineral content ratio [11]. More studies are needed to confirm which is the best measurement tool for obese patients.

Previous studies found that overall prevalence of overweight/obesity was higher in females than in males, and the prevalence of central obesity was also higher in females than in males [53]. Intraocular pressure changes may be linked to metabolic syndrome in postmenopausal women, but not in premenopausal women $[54,55]$. Because men and women have such different body compositions, in this study, the data were stratified according to sex. The results revealed that the anthropometric parameters of adiposity were associated with IOP, especially among women. Estrogen may also play an important role, and ESR2 gene polymorphism is considered to be associated with elevated IOP in female patients with OAG [56]. Estrogen can regulate smooth muscle tone and vascular resistance and, as a result, augments the activity of endothelial-based nitric oxide synthase and has a hypertensive effect by influencing aqueous production and outflow systems [57]. Gimeno and Klaman [58] suggested that adipose tissue may serve as an endocrine organ and secrete other paracrine factors that could also influence retinal ganglion cell health. Nevertheless, the underlying mechanisms between obesity, sex, and OAG are unclear.

However, several potential limitations should be taken into account in the interpretation of these findings. First, the main limitation is that only five studies were prospective and the others were retrospective. There was no standard method to assess the quality of the included studies, and substantial heterogeneity was found in the methods and quality of the original studies. Significant variability was found in terms of the study design, exclusion criteria, inclusion criteria, definition of exposure and outcomes, method of diagnosis of glaucoma, IOP measurement, and the difference between anthropometric-parameter measurements for adiposity. For instance, abdominal adiposity was measured by WC or WHR, while general adiposity was measured by BMI. Begg's funnel plot showed a little asymmetry because of the heterogeneity between studies. All the aforementioned factors could be the source of the high heterogeneity between the studies.

Second, the heterogeneity of uncontrolled or residual confounding from different original studies may influence the present study's results. The more components of metabolic syndrome a person has, the higher the risk of OAG. Metabolic-syndrome components including obesity, hypertension, diabetes mellitus, and hyperlipidemia may play a role in the pathogenesis of OAG $[24,25,30]$. Not only for hypertension [59] and diabetes mellitus, another metaanalysis also found that obstructive sleep apnea syndrome has a relationship with elevated IOP and the relevance of glaucoma [15]. Moreover, obese patients almost always combined the above systematic factors with bad habits such as alcohol consumption, cigarette smoking, and little activity [60]. Despite using the random-effects model to summarize the pooled estimate data in order to minimize the effects of heterogeneous variability between studies, the residual or unmeasured confounding factors may affect the results of the present study.

Third, potential selection bias is likely to exist. Different studies with different cases and control participants resulted in selection bias because different ophthalmologists would not have used exactly the same criteria. In addition, studies were limited to the English language, which may mean that data were missed from important studies published in other languages. Finally, despite the comprehensive search of all relative articles, many articles did not meet the inclusion criteria. Publication bias was still inevitable because research with null results was not reported. 


\section{Conclusions}

In the meta-analysis, it was found that adiposity has a higher risk of elevated IOP and abdominal adiposity has a positive association with glaucoma, especially in female patients. Future research should focus on longitudinal cohort studies with objective measurements for adiposity and should consider the mechanisms for obesity and the risk of glaucoma. Understanding the systematic factors associated with elevated IOP and OAG progression will help clinicians in screening and monitoring patients in the early stages.

\section{Conflicts of Interest}

No conflicting relationship exists for any author.

\section{Authors' Contributions}

Weiming Liu, Jiawen Ling, and Peirong Lu conceived of the idea and designed the study. Weiming Liu and Yiyi Chen collected the data. Weiming Liu and Yan Wu performed the data analysis. Weiming Liu, Jiawen Ling, and Peirong $\mathrm{Lu}$ participated in the critical revision of the manuscript. All authors read and approved the final manuscript. The first two authors contributed equally as first authors.

\section{Acknowledgments}

This study was supported in part by the Jiangsu Province's Key Provincial Talents Program (no. RC2011104) and the Soochow Scholar Project of Soochow University.

\section{References}

[1] H. A. Quigley and A. T. Broman, "The number of people with glaucoma worldwide in 2010 and 2020," The British Journal of Ophthalmology, vol. 90, no. 3, pp. 262-267, 2006.

[2] M. C. Leske, A. M. Connell, S. Y. Wu, L. G. Hyman, and A. P. Schachat, "Risk factors for open-angle glaucoma. The Barbados Eye Study," Archives of Ophthalmology, vol. 113, no. 7, pp. 918-924, 1995.

[3] M. Zhou, W. Wang, W. Huang, and X. Zhang, "Diabetes mellitus as a risk factor for open-angle glaucoma: a systematic review and meta-analysis," PloS One, vol. 9, no. 8, article e102972, 2014.

[4] S. M. Chiotoroiu, D. Pop de Popa, G. I. Stefaniu, F. A. Secureanu, and V. L. Purcarea, "The importance of alcohol abuse and smoking in the evolution of glaucoma disease," Journal of Medicine and Life, vol. 6, no. 2, pp. 226-229, 2013.

[5] J. H. Kang, J. L. Wiggs, B. A. Rosner, J. Haines, W. Abdrabou, and L. R. Pasquale, "Endothelial nitric oxide synthase gene variants and primary open-angle glaucoma: interactions with hypertension, alcohol intake, and cigarette smoking," Archives of Ophthalmology, vol. 129, no. 6, pp. 773-780, 2011.

[6] Obesity: preventing and managing the global epidemic. Report of a WHO consultation," World Health Organization Technical Report Series, vol. 894, p. i-xii, 1-253, 2000.

[7] L. A. Wise, L. Rosenberg, R. G. Radin et al., "A prospective study of diabetes, lifestyle factors, and glaucoma among African-American women," Annals of Epidemiology, vol. 21, no. 6, pp. 430-439, 2011.
[8] E. Cohen, M. Kramer, T. Shochat, E. Goldberg, M. Garty, and I. Krause, "Relationship between body mass index and intraocular pressure in men and women: a population-based study," Journal of Glaucoma, vol. 25, no. 5, pp. e509-e513, 2016.

[9] S. Ngo, A. Harris, B. A. Siesky, A. Schroeder, G. Eckert, and S. Holland, "Blood pressure, ocular perfusion pressure, and body mass index in glaucoma patients," European Journal of Ophthalmology, vol. 23, no. 5, pp. 664-669, 2013.

[10] H. D. Jang, D. H. Kim, K. Han et al., "Relationship between intraocular pressure and parameters of obesity in Korean adults: the 2008-2010 Korea National Health and Nutrition Examination Survey," Current eye Research, vol. 40, no. 10, pp. 1008-1017, 2015.

[11] H. T. Kim, J. M. Kim, J. H. Kim et al., "Relationships between anthropometric measurements and intraocular pressure: the Korea National Health and Nutrition Examination Survey," American Journal of Ophthalmology, vol. 173, pp. 23-33, 2017.

[12] D. Moher, A. Liberati, J. Tetzlaff, and D. G. Altman, "Preferred reporting items for systematic reviews and meta-analyses: the PRISMA statement," BMJ, vol. 339, p. b2535, 2009.

[13] D. F. Stroup, J. A. Berlin, S. C. Morton et al., "Meta-analysis of observational studies in epidemiology: a proposal for reporting. Meta-analysis of observational studies in epidemiology (MOOSE) group," Jama, vol. 283, no. 15, pp. 20082012, 2000.

[14] E. von Elm, D. G. Altman, M. Egger, S. J. Pocock, P. C. Gotzsche, and J. P. Vandenbroucke, "The strengthening the reporting of observational studies in epidemiology (STROBE) statement: guidelines for reporting observational studies," Journal of Clinical Epidemiology, vol. 61, no. 4, pp. 344-349, 2008.

[15] Y. Shi, P. Liu, J. Guan, Y. Lu, and K. Su, “Association between glaucoma and obstructive sleep apnea syndrome: a metaanalysis and systematic review," PloS One, vol. 10, no. 2, article e0115625, 2015.

[16] Y. H. Cui, C. X. Jing, and H. W. Pan, “Association of blood antioxidants and vitamins with risk of age-related cataract: a meta-analysis of observational studies," The American Journal of Clinical Nutrition, vol. 98, no. 3, pp. 778-786, 2013.

[17] J. J. Gagne and M. C. Power, "Anti-inflammatory drugs and risk of Parkinson disease: a meta-analysis," Neurology, vol. 74, no. 12, pp. 995-1002, 2010.

[18] R. DerSimonian and N. Laird, "Meta-analysis in clinical trials," Controlled Clinical Trials, vol. 7, no. 3, pp. 177-188, 1986.

[19] C. B. Begg and M. Mazumdar, "Operating characteristics of a rank correlation test for publication bias," Biometrics, vol. 50, no. 4, pp. 1088-1101, 1994.

[20] M. Egger, G. Davey Smith, M. Schneider, and C. Minder, "Bias in meta-analysis detected by a simple, graphical test," $B M J$, vol. 315, no. 7109, pp. 629-634, 1997.

[21] F. Kyari, M. M. Abdull, R. Wormald et al., "Risk factors for open-angle glaucoma in Nigeria: results from the Nigeria National Blindness and Visual Impairment Survey," BMC Ophthalmology, vol. 16, p. 78, 2016.

[22] F. Ko, M. V. Boland, P. Gupta et al., "Diabetes, triglyceride levels, and other risk factors for glaucoma in the National Health and Nutrition Examination Survey 2005-2008," Investigative Ophthalmology \& Visual Science, vol. 57, no. 4, pp. 2152-2157, 2016. 
[23] G. S. Tan, T. Y. Wong, C. W. Fong, and T. Aung, "Diabetes, metabolic abnormalities, and glaucoma," Archives of Ophthalmology, vol. 127, no. 10, pp. 1354-1361, 2009.

[24] M. Kim, J. W. Jeoung, K. H. Park, W. H. Oh, H. J. Choi, and D. M. Kim, "Metabolic syndrome as a risk factor in normaltension glaucoma," Acta Ophthalmologica, vol. 92, no. 8, pp. e637-e643, 2014.

[25] K. Imai, M. Hamaguchi, K. Mori et al., "Metabolic syndrome as a risk factor for high-ocular tension," International Journal of Obesity, vol. 34, no. 7, pp. 1209-1217, 2010.

[26] S. A. Rasoulinejad, A. Kasiri, M. Montazeri et al., "The association between primary open angle glaucoma and clustered components of metabolic syndrome," The Open Ophthalmology Journal, vol. 9, pp. 149-155, 2015.

[27] F. Aptel, C. Chiquet, R. Tamisier et al., “Association between glaucoma and sleep apnea in a large French multicenter prospective cohort," Sleep Medicine, vol. 15, no. 5, pp. 576-581, 2014.

[28] X. Jiang, R. Varma, S. Wu et al., "Baseline risk factors that predict the development of open-angle glaucoma in a population: the Los Angeles Latino eye study," Ophthalmology, vol. 119, no. 11, pp. 2245-2253, 2012.

[29] L. R. Pasquale, W. C. Willett, B. A. Rosner, and J. H. Kang, "Anthropometric measures and their relation to incident primary open-angle glaucoma," Ophthalmology, vol. 117, no. 8, pp. 1521-1529, 2010.

[30] P. A. Newman-Casey, N. Talwar, B. Nan, D. C. Musch, and J. D. Stein, "The relationship between components of metabolic syndrome and open-angle glaucoma," Ophthalmology, vol. 118, no. 7, pp. 1318-1326, 2011.

[31] J. B. Jonas, R. Ritch, and S. Panda-Jonas, "Cerebrospinal fluid pressure in the pathogenesis of glaucoma," Progress in Brain Research, vol. 221, pp. 33-47, 2015.

[32] J. B. Jonas, N. Wang, Y. X. Wang et al., "Body height, estimated cerebrospinal fluid pressure and open-angle glaucoma. The Beijing Eye Study 2011," PloS One, vol. 9, no. 1, article e86678, 2014.

[33] L. Xu, Y. X. Wang, S. Wang, and J. B. Jonas, "Neuroretinal rim area and body mass index," PloS One, vol. 7, no. 1, article e30104, 2012.

[34] J. B. Jonas, V. Nangia, R. Gupta et al., "Retinal nerve fibre layer cross-sectional area, neuroretinal rim area and body mass index," Acta Ophthalmologica, vol. 92, no. 3, pp. e194-e199, 2014.

[35] Y. Zheng, C. Y. Cheung, T. Y. Wong, P. Mitchell, and T. Aung, "Influence of height, weight, and body mass index on optic disc parameters," Investigative Ophthalmology \& Visual Science, vol. 51, no. 6, pp. 2998-3002, 2010.

[36] J. P. Berdahl, D. Fleischman, J. Zaydlarova, S. Stinnett, R. R. Allingham, and M. P. Fautsch, "Body mass index has a linear relationship with cerebrospinal fluid pressure," Investigative Ophthalmology \& Visual Science, vol. 53, no. 3, pp. 14221427, 2012.

[37] Z. Onaran, O. Konuk, S. O. Oktar, C. Yucel, and M. Unal, "Intraocular pressure lowering effect of orbital decompression is related to increased venous outflow in Graves orbitopathy," Current eye Research, vol. 39, no. 7, pp. 666-672, 2014.

[38] N. Wang, X. Xie, D. Yang et al., "Orbital cerebrospinal fluid space in glaucoma: the Beijing intracranial and intraocular pressure (iCOP) study," Ophthalmology, vol. 119, no. 10, pp. 2065-2073, 2012, e1.
[39] C. J. Bulpitt, C. Hodes, and M. G. Everitt, "Intraocular pressure and systemic blood pressure in the elderly," The British Journal of Ophthalmology, vol. 59, no. 12, pp. 717-720, 1975.

[40] A. Gunes, F. Uzun, E. E. Karaca, and M. Kalayci, "Evaluation of anterior segment parameters in obesity," Korean Journal of Ophthalmology, vol. 29, no. 4, pp. 220-225, 2015.

[41] A. Bouloumie, T. Marumo, M. Lafontan, and R. Busse, "Leptin induces oxidative stress in human endothelial cells," The FASEB Journal, vol. 13, no. 10, pp. 1231-1238, 1999.

[42] S. C. Sacca, A. Pascotto, P. Camicione, P. Capris, and A. Izzotti, "Oxidative DNA damage in the human trabecular meshwork: clinical correlation in patients with primary open-angle glaucoma," Archives of Ophthalmology, vol. 123, no. 4, pp. 458-463, 2005.

[43] D. Risner, R. Ehrlich, N. S. Kheradiya, B. Siesky, L. McCranor, and A. Harris, "Effects of exercise on intraocular pressure and ocular blood flow: a review," Journal of Glaucoma, vol. 18, no. 6, pp. 429-436, 2009.

[44] J. Gale, A. P. Wells, and G. Wilson, "Effects of exercise on ocular physiology and disease," Survey of Ophthalmology, vol. 54, no. 3, pp. 349-355, 2009.

[45] M. G. dos Santos, S. Makk, A. Berghold, M. Eckhardt, and A. Haas, "Intraocular pressure difference in Goldmann applanation tonometry versus Perkins hand-held applanation tonometry in overweight patients," Ophthalmology, vol. 105, no. 12, pp. 2260-2263, 1998.

[46] A. K. Lam and C. H. Lam, "Effect of breath-holding on pulsatile ocular blood flow measurement in normal subjects," Optometry and Vision Science, vol. 81, no. 8, pp. 597-600, 2004.

[47] D. A. Levine, D. A. Calhoun, R. J. Prineas, M. Cushman, V. J. Howard, and G. Howard, "Moderate waist circumference and hypertension prevalence: the REGARDS study," American Journal of Hypertension, vol. 24, no. 4, pp. 482-488, 2011.

[48] K. G. Alberti, P. Zimmet, and J. Shaw, "The metabolic syndrome-a new worldwide definition," Lancet, vol. 366, no. 9491, pp. 1059-1062, 2005.

[49] J. P. Despres and I. Lemieux, "Abdominal obesity and metabolic syndrome," Nature, vol. 444, no. 7121, pp. 881-887, 2006.

[50] S. D. Hsieh and H. Yoshinaga, "Abdominal fat distribution and coronary heart disease risk factors in men-waist/height ratio as a simple and useful predictor," International Journal of Obesity and Related Metabolic Disorders, vol. 19, no. 8, pp. 585-589, 1995.

[51] G. Vazquez, S. Duval, D. R. Jacobs Jr., and K. Silventoinen, "Comparison of body mass index, waist circumference, and waist/hip ratio in predicting incident diabetes: a meta-analysis," Epidemiologic Reviews, vol. 29, pp. 115-128, 2007.

[52] P. Srikanthan, T. E. Seeman, and A. S. Karlamangla, "Waist-hip-ratio as a predictor of all-cause mortality in high-functioning older adults," Annals of Epidemiology, vol. 19, no. 10, pp. 724-731, 2009.

[53] L. Wang, J. Lyu, Y. Guo et al., "Regional specific differences in prevalence of overweight/obesity in China: findings from China Kadoorie Biobank study in 10 areas in China," Zhonghua Liu Xing Bing Xue Za Zhi, vol. 36, no. 11, pp. 1190-1194, 2015.

[54] B. J. Park, J. O. Park, H. T. Kang, and Y. J. Lee, “Elevated intraocular pressure is associated with metabolic syndrome 
in postmenopausal women: the Korean National Health and Nutrition Examination Survey," Menopause, vol. 20, no. 7, pp. 742-746, 2013.

[55] R. N. Khurana, L. D. LaBree, G. Scott, R. E. Smith, and S. C. Yiu, "Esterified estrogens combined with methyltestosterone raise intraocular pressure in postmenopausal women," American Journal of Ophthalmology, vol. 142, no. 3, pp. 494-495, 2006.

[56] F. Mabuchi, Y. Sakurada, K. Kashiwagi, Z. Yamagata, H. Iijima, and S. Tsukahara, "Estrogen receptor beta gene polymorphism and intraocular pressure elevation in female patients with primary open-angle glaucoma," American Journal of Ophthalmology, vol. 149, no. 5, pp. 826-830, 2010, e1-2.

[57] T. S. Vajaranant and L. R. Pasquale, "Estrogen deficiency accelerates aging of the optic nerve," Menopause, vol. 19, no. 8, pp. 942-947, 2012.

[58] R. E. Gimeno and L. D. Klaman, "Adipose tissue as an active endocrine organ: recent advances," Current Opinion in Pharmacology, vol. 5, no. 2, pp. 122-128, 2005.

[59] H. W. Bae, N. Lee, H. S. Lee, S. Hong, G. J. Seong, and C. Y. Kim, "Systemic hypertension as a risk factor for open-angle glaucoma: a meta-analysis of population-based studies," PloS One, vol. 9, no. 9, article e108226, 2014.

[60] G. Roddy, D. Curnier, and D. Ellemberg, "Reductions in intraocular pressure after acute aerobic exercise: a meta-analysis," Clinical Journal of Sport Medicine, vol. 24, no. 5, pp. 364372, 2014. 


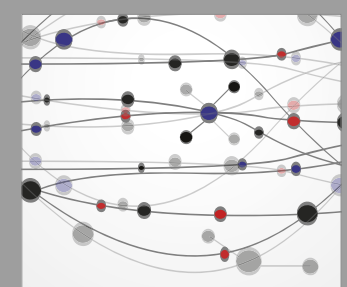

The Scientific World Journal
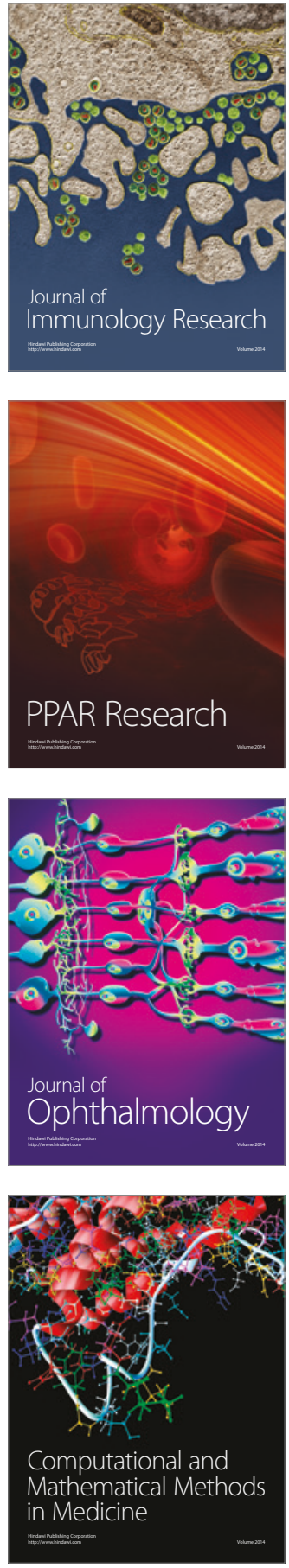

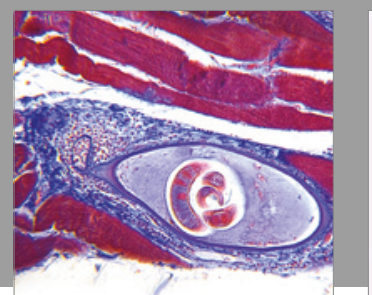

Gastroenterology Research and Practice
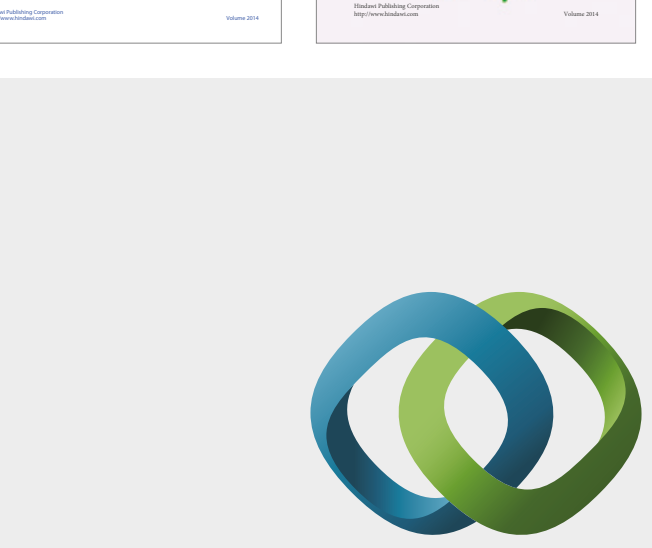

\section{Hindawi}

Submit your manuscripts at

https://www.hindawi.com
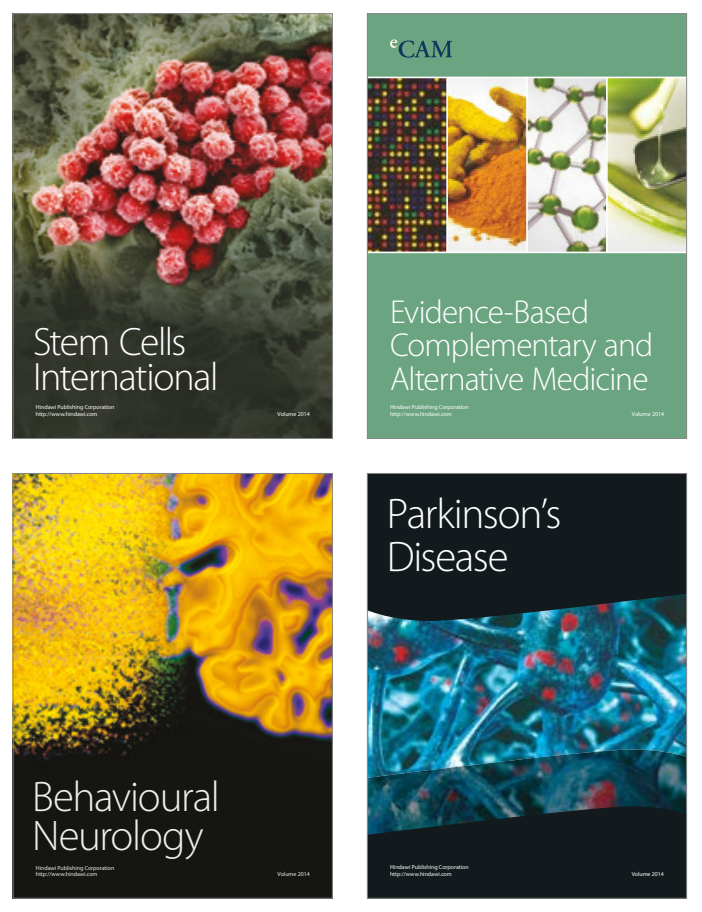
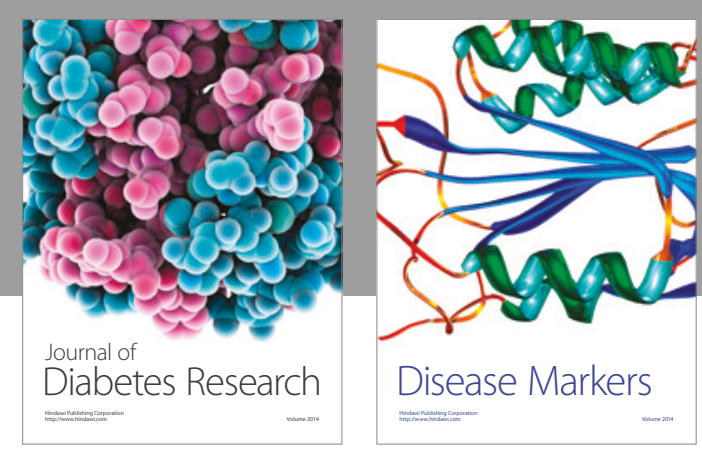

Disease Markers
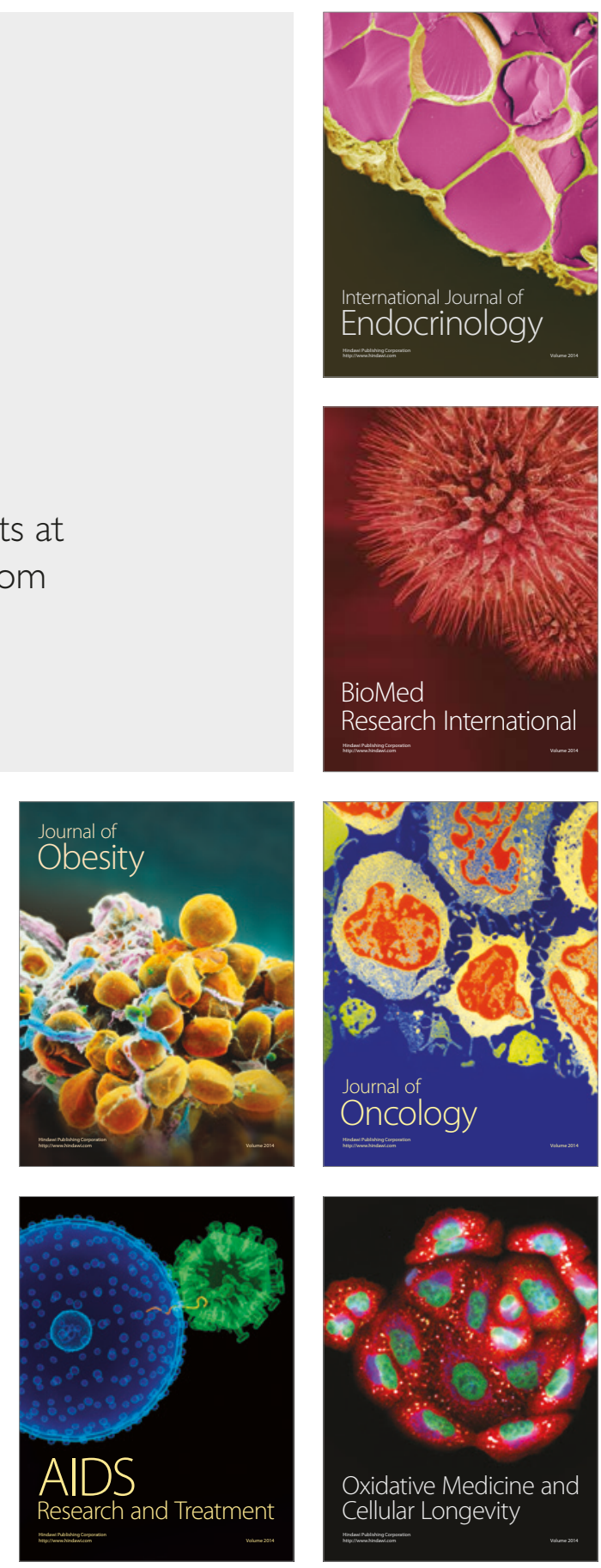